moderators was carried out and themes were developed based on appropriate and relevant $\mathrm{MeSH}$ terms in order to create the categories and items of the clinical inventory.

Result. Seventy-three different tic severity moderators were identified, the most common being exercise, sleep, peer victimisation, psychosocial stress, watching TV, academic activities and distraction. Twenty-nine themes emerged from the thematic analysis which were then used to update the clinical inventory of tic severity moderators. The review also highlighted the subjectivity of these moderators' effects on tic severity as some moderators were tic-worsening in some individuals and tic-improving or neutral in others, which is contrary to the current dichotomous understanding.

Conclusion. The updated clinical inventory of tic severity moderators invites researchers and clinicians to be more aware of the existence, variability and subjective effects of these tic severity moderators in individuals with TS, as these have been previously looked in a dichotomous way. By better identifying tic-severity moderators and their worsening, improving or neutral effects on tic severity this clinician rated inventory will have potentially important, direct implications for the management and treatment of tics.

\section{Differentiated white blood cell count measures as a predictor of weight in anorexia nervosa}

\author{
Benjamin Cross $^{1 \star}$ and Matthew Cahill ${ }^{2}$ \\ ${ }^{1}$ East Lancashire Hospitals NHS Trust and ${ }^{2}$ Cheshire \& Wirral \\ Partnership NHS Foundation Trust \\ ${ }^{\star}$ Corresponding author.
}

doi: 10.1192/bjo.2021.657

Aims. To explore the relationship between weight, BMI and differentiated white cell count in patients with anorexia nervosa that have commenced refeeding protocols.

Hypotheses:

1. Weight and BMI will be positively correlated with differentiated white blood cell measures.

2. Regression analyses will indicate that differentiated white blood cell measures can predict weight and BMI.

Background. Anorexia nervosa is a serious mental illness with the highest psychiatric mortality rate. It is characterised by an extreme overvaluation of body image and a preoccupation with weight loss that is achieved through calorie restriction, purging or laxative abuse. The eventual malnutrition that is as a result of self-starvation causes significant negative physical sequelae. One such consequence is the detrimental effect that starvation has on the immune system, leading to bone marrow suppression and cytopenias. In obesity, weight loss has been shown to cause reductions in differentiated white blood cell counts, which are often reduced in anorexia. No studies to date have explored whether weight is related to white blood cell count in anorexia, despite being a common finding in patients.

Method. All subjects were inpatients or ex-inpatients on an eating disorder unit in the Wirral, NW England. Subjects were assessed according to an inclusion criterion. 12 consecutive weekly weights, BMIs and differentiated white blood cell measures were collected for analysis in SPSS. Correlational and regression analyses were then performed to examine the relationship between variables.

Result. 302 data points from 26 individuals were used in the final analysis. Weight and BMI both moderately positively correlated with white cell count, $\mathrm{r}=0.364 \mathrm{p}<0.001 \mathrm{~N}=302$ and $\mathrm{r}=0.521$ $\mathrm{p}<0.001 \mathrm{~N}=302$, respectively. Weight and BMI also moderately positively correlated with all differentiated measures of white blood cell count. Linear regression revealed a significant regression equation between weight, $\mathrm{BMI}$ and white blood cell count: $(\mathrm{F}(1,301)=22.482, \mathrm{p}<0.001) \mathrm{R} 2=0.69$ and $(\mathrm{F}(1,301)=$ $112.244 \mathrm{p}<0.001) \mathrm{R} 2=0.272$, respectively.

Conclusion. We have demonstrated that there is a moderately positive relationship between weight, BMI and differentiated white blood cell measures. Furthermore, in line with our hypotheses, differentiated white blood cell measures have some power to predict weight and BMI. This further implicates the effect that starvation and anorexia nervosa have on the immune system. It also highlights the possibility that it might be possible to use immunological markers in order to deduce whether weights have been falsified by those with anorexia nervosa.

The impact of the March 2020 lockdown on the cardiometabolic risk factors of male forensic and rehabilitation patients

Nicholas Dodough*, Jaspreet Phull, Jaswant Singh and Jackie Sendell

Lincolnshire Partnership Foundation Trust

${ }^{\star}$ Corresponding author.

doi: 10.1192/bjo.2021.658

Aims. To explore the impact of the March 2020 lockdown restrictions on the cardiometabolic risk factors of male forensic and rehabilitation inpatients in one NHS trust in the United Kingdom. Method. Retrospective data from September 2019 to September 2020 (six months before and after the 23 March 2020 lockdown) was collected by evaluating the health records of male patients in a low secure forensic ward and two rehabilitation units.

Result. The number of patients with BMI values within the study period was 34 while the number of patients with blood results was 26. This study showed that the average BMI six months before the start of the March lockdown was $29.8 \mathrm{~kg} / \mathrm{m} 2$ while the average $\mathrm{BMI}$ at the end of six months after the lockdown was $31.8 \mathrm{~kg} / \mathrm{m} 2$.

The results from the 6-month interval before the March 2020 lockdown $(\mathrm{M}=0, \mathrm{SD}=0)$ and the 6-month interval after the March 2020 lockdown $(\mathrm{M}=0.9, \mathrm{SD}=4.16)$ indicate that the March 2020 lockdown resulted in an increase of BMI, $\mathrm{t}(5)=$ $2.42, \mathrm{P}=0.036$. The result is significant at $\mathrm{p}<0.05$

$8.8 \%$ of patients had an increase in their doses of antihypertensive agents after the lockdown whereas no patients had an increase of dose before the lockdown. $7.7 \%$ of patients had an HBAlc of more than $48 \mathrm{mmol} / \mathrm{L}$ after the lockdown compared to $3.8 \%$ before the lockdown. The serum triglycerides and total cholesterol levels also increased after the lockdown with an average increase of $0.17 \mathrm{mmol} / \mathrm{L}$ and $0.25 \mathrm{mmol} / \mathrm{L}$ respectively. The average serum HDL levels decreased after the lockdown with an average decrease of $0.06 \mathrm{mmol} / \mathrm{L}$.

Conclusion. There appears to be a positive correlation between the onset of the March 2020 lockdown and an increase of BMI, worsening of blood pressure, glycemic control and lipid metabolism. Limitations

(1) Waist circumference was not measured during the study period preventing us from examining all of the features of metabolic syndrome.

(2) This study did not look at the levels of physical activity (such as access to section 17 leave) and dietary habits before and after the March 2020 lockdown which may explain the results found. 